\title{
On the Vafa-Witten Theorem on Spontaneous Breaking of Parity
}

\author{
Martin B. Einhorn \\ Michigan Center for Theoretical Physics, \\ Randall Laboratory, The University of Michigan, \\ Ann Arbor, MI 48109 \\ E-mail: meinhorn@umich.ed \\ José Wudka \\ Department of Physics \\ University of California, Riverside CA 92521-0413, USA \\ E-mail: Jose.Wudka@cur.ed \\ (Dated: November 3, 2018)
}

\begin{abstract}
We revisit the proof provided by Vafa and Witten that there is no spontaneous parity breaking in theories with vector-like fermions. We argue that the various criticisms that have been leveled at the original proof do not invalidate it
\end{abstract}

PACS numbers: 11.30.-j 11.30.Er 11.30.Qc

\section{INTRODUCTION}

Several years ago, Vafa and Witten[1] (hereafter referred to as VW) provided a simple, elegant argument for the absence of spontaneous parity violation in theories containing only vector-like fermions; this result, however, has been criticized on various grounds dealing with mathematical niceties underlying the proof. It was argued in [2] and later in [3] that the expression for the free energy on which the VW argument is based has an ill-defined infinite-volume limit and is therefore insufficient to determine the absence of spontaneous parity violation. Previously, Aoki[4] had noted the possibility that lattice QCD has a parity-violating phase which disappears in the continuum limit; this apparently presents a problem for the VW result since the original proof can be applied to the lattice-regulated theory as well as to the continuum. Hence the Aoki phase, even if only present at non-zero lattice spacing, would directly contradict the results of [1] provided the assumptions made by VW are satisfied by the lattice regulated theory at finite spacing. This model was studied carefully by Sharpe and Singleton[5] who argued that the Aoki phase is not ruled out by the results of VW since their arguments appear not to be applicable for the case of fermionic order parameters. Finally, reference [6] raised the possibility of parity-violating operators that evade the proof of VW for systems in a thermal bath. In this paper, we will re-examine these complaints and find that, for physically relevant systems, they do not invalidate the results of VW.[1]In a related paper,[7] we argue that the proof, even under the assumptions stated, does not however exclude spontaneous parity violation under certain circumstances.

We organize this paper as follows. In section II we review the original VW argument. In section III we examine the large volume limit of the theory and show that, as claimed in [2], it is well defined only for the case where spontaneous symmetry breaking does not occur; yet we will argue that this deficiency does not invalidate the results of VW. We revisit the lattice arguments in section IV where we argue that the Aoki phases (at least for a large number of fermions) do not represent absolute minima of the effective potential but only metastable states ${ }^{1}$. In section $\mathrm{V}$ we consider the use of fermionic operators as order parameters for a possible parity-violating phase transition; in contrast to the results of [5] we find that this type of order parameter exhibits the same behavior claimed by VW. We then argue that there is a simple and useful fermionic operator that can be better used as an order parameter since it preserves chirality (the operators used in $[4,5]$ violate both parity and chiral symmetry). Parity violation at finite temperature is briefly revisited in Sect. VI, and parting comments are presented in section VII. A mathematical result is relegated to the Appendix.

\footnotetext{
1 This should not be used to completely dismiss these states as irrelevant: recent experiments in heavy-ion collisions create finite-volume systems that may relax temporarily into parity-violating configurations[8]
} 


\section{THE VAFA-WITTEN ARGUMENT}

In this section we summarize the original VW argument. The idea is to consider a theory with action $S$ that is even under parity transformations and contains only vector-like fermions (so that the corresponding fermionic determinant is real), and to add a parity-violating term $X$ so that the full Minkowski-space action becomes

$$
S_{\mathrm{tot}}=S+\lambda X
$$

where $\lambda$ is a small real parameter and $X$ is Hermitian. We will assume (without loss of generality) that $X$ is parity odd; all gauge-fixing terms are assumed to be contained in $S$. The bosonic terms in $S$ are assumed to be real (this is always possible for all linear gauges) ${ }^{2}$, (see for example [9]). We will concentrate on theories that do not contain scalar fields.

We first consider the case where $X$ is a purely bosonic operator. Some comments concerning scalar theories are provided in section VII. We first obtain the free energy by Wick-rotating $S$ in (1). While not without ambiguities, to be precise, we will follow the procedure of [10]. Any parity-violating operator contains an $\epsilon$ tensor and this implies that the corresponding term acquires an additional factor of $i$ upon Wick rotation, thus we find

$$
i S_{\mathrm{tot}} \rightarrow-S_{E}+i \lambda X_{E}
$$

with $S_{E}$ and $X_{E}$ real (the subscript $E$ will be used to denote the quantities in Euclidean space). The "free energy" $W$ is then

$$
e^{-W(\lambda)}=\int[d A] e^{-S_{E}^{\text {boson }}+i \lambda X_{E}} \operatorname{Det}\left(\not D_{E}+M\right)
$$

where $A$ denotes the gauge fields, and $S_{E}^{\text {boson }}$ the purely bosonic contributions to $S_{E} \cdot{ }^{3}$ We take the mass matrix $M$ diagonal with non-negative eigenvalues (as we are not interested in explicit $\mathrm{P}$ violation); the Euclidean-space fermionic covariant derivative is denoted by $D_{E}$ which is anti-Hermitian in our conventions [10] (the gamma matrices are Hermitian). The fermionic determinant is then a real functional of the gauge fields for vector-like couplings[11].

Consider now the case where $X$ is a fermionic bilinear ${ }^{4}$,

$$
X=\bar{\psi} \mathcal{O} \psi
$$

After a Wick rotation and to first order in $\lambda$ the fermionic integration yields

$$
e^{-W(\lambda)}=\int[d A] e^{-S_{E}^{\text {boson }}+i \lambda X_{E}^{(\text {eff })}} \operatorname{Det}\left(\not D_{E}+M\right) ; \quad X_{E}^{(\text {eff })}=-i \operatorname{Tr}\left[\frac{1}{\not D_{E}+M} \mathcal{O}_{E}\right]
$$

where $\mathcal{O}_{E}=\gamma^{0} \gamma^{5} \mathcal{O} \gamma^{5} \gamma^{0}{ }^{5}$. A naive calculation shows that $X_{E}^{(\text {eff })}$ is real if $\mathcal{O}_{E}^{\dagger}=-\gamma^{5} \mathcal{O}_{E} \gamma^{5}$. In particular note that this holds for the simple cases $\mathcal{O}_{E}=i \gamma_{5}$ and $\mathcal{O}_{E}=\overleftrightarrow{\not D}_{E} \gamma^{5}$. There is, of course, a possibility that these rather formal results are flawed by the mathematical subtleties involved in properly defining $X_{E}^{(\text {eff })}[5]$; we discuss this possibility it in section $\mathrm{V}$.

With these preliminaries the proof is based on the claim [1] that all parity-violating operators correspond to real $X_{E}$ or $X_{E}^{(\text {eff })}$, in which case

$$
W(\lambda) \geq W(0)
$$

as a result of Schwartz's inequality. The validity of this result then rests on the requirement that the measure is positive definite, that is, $\operatorname{Det}\left(\not D_{E}+M\right)>0$. This is true for the case of vector-like fermions [13], but can be extended to some more general types of fermionic Lagrangians; we return to this point in section IV. Note that in proving (6)

\footnotetext{
${ }^{2}$ For this it is convenient not to introduce ghosts and deal with the gauge-fixing determinant directly.

${ }^{3}$ For constant $\lambda, W(\lambda)$ will be proportional to the space-time volume.

${ }^{4}$ When $X$ contains products of bilinears these can be turned into a sum of bilinears through the introduction of appropriate auxiliary fields by using the Hubbard-Stratonovich trick [12]; the arguments below carry through provided these auxiliary fields are vectors; this will be investigated in detail in [7].

${ }^{5}$ It is understood that $\mathcal{O}_{E}$ is obtained by replacing $D_{0} \rightarrow-i D_{0}^{E}, \gamma^{0} \rightarrow \gamma_{0}^{E}, \gamma^{k} \rightarrow-i \gamma_{k}^{E}$ in $\mathcal{O}$. In our conventions $\gamma_{\mu}^{E \dagger}=+\gamma_{\mu}^{E}$ and $\left\{\gamma_{\mu}^{E}, \gamma_{\nu}^{E}\right\}=\delta_{\mu \nu}$
} 
we used the fact that $W$ is real since $X$ is odd under parity and the integration involves both a configuration and its parity conjugate.

Since any explicit violation of the symmetry (in this case parity) results in an increased free energy, the lowest energy ground state will be parity symmetric. To see that eq. (6) implies vanishing of the expectation value of $X, \bar{X}$, it is useful to pass to the effective potential $V(\bar{X})$

$$
V(\bar{X}) \equiv W(\lambda)-\lambda \bar{X}, \text { with } \bar{X} \equiv \frac{\partial W}{\partial \lambda} .
$$

Inversely, using $\lambda=-V^{\prime}(\bar{X})$, we can write this as

$$
W(\lambda)=V(\bar{X})+\lambda \bar{X}=V(\bar{X})-\bar{X} V^{\prime}(\bar{X}) .
$$

We want to show that, in the limit $\lambda \rightarrow 0^{ \pm}, \bar{X} \rightarrow 0$. Suppose, on the contrary, that ${ }^{6}$ as $\lambda \rightarrow 0^{+}, \bar{X}=v \neq 0$. This is necessarily at a minimum of $V(\bar{X}) \cdot{ }^{7}$ If we adopt the usual convention that $W(0)=0$, then $V(v)=0$ as well. The inequality eq. (6) therefore implies that

$$
V(\bar{X})-\bar{X} V^{\prime}(\bar{X})=\left\{V(\bar{X})-(\bar{X}-v) V^{\prime}(\bar{X})\right\}-v V^{\prime}(\bar{X}) \geq 0 .
$$

Since $v$ is a minimum, $V^{\prime}(v)=0$. If we expand in a neighborhood of $\bar{X}=v$, the term in the curly brackets contains at least one power of $(\bar{X}-v)$ greater than the last term and, moreover, since $v$ is a minimum, the leading term in the expansion of $V^{\prime}(\bar{X})$ will be an odd power of $(\bar{X}-v)$. (For $V^{\prime \prime}(v)>0$, it will be linear in $(\bar{X}-v)$.) But then the inequality will be violated in a neighborhood of $\bar{X}=v$. Therefore, the only possibility is that $v=0 .{ }^{8}$.

The same conclusion can be obtained starting form the Hamiltonian derived form (1) ${ }^{9}$. Indeed, if the ground state is not parity symmetric, then, when $\lambda=0$, there will be two degenerate lowest-energy states $| \pm\rangle$ that are parity conjugate of each other. Now, the Hamiltonian corresponding to (1) is of the form $H+\lambda \Xi$ where $H$ is parity even and $\Xi$ parity odd; a straightforward application of degenerate perturbation theory shows that the term $\lambda \Xi$ lifts the degeneracy, so that the lowest energy state for the system equals

$$
E_{0}-|\lambda \epsilon|+O\left(\lambda^{2}\right) ; \quad E_{0}=\langle \pm|H| \pm\rangle ; \epsilon=\langle+|\Xi|-\rangle
$$

that decreases as a function of $\lambda$. This contradicts (6) and implies that there are no parity-degenerate vacua: the symmetry is not broken.

\section{LARGE VOLUME EFFECTS}

The results summarized in the previous section were criticized in [2] based on the following observation. Suppose we construct a Landau-Ginzburg functional of the order parameter defined as $X_{E} / V$, where $V$ denotes the spatial volume of the system. Explicitly,

$$
\begin{aligned}
e^{-W} & =\int_{-\infty}^{\infty} d \rho \int[d A] e^{-S_{E}^{\text {boson }}+i \lambda X_{E}} \operatorname{Det}\left(\not D_{E}+M\right) \delta\left(\rho-X_{E} / V\right), \\
& =\int_{-\infty}^{\infty} d \rho e^{-\mathcal{F}(\rho)+i V \lambda \rho}
\end{aligned}
$$

where $\mathcal{F}$ is presumably proportional to $V$ and we assume it can be expanded in a power series in $\rho$. Since the original action is parity symmetric $\mathcal{F}$ will be even in $\rho$. For definiteness we will consider the case

$$
\mathcal{F}(\rho)=V\left[\frac{1}{4} b \rho^{4}+\frac{1}{2} a \rho^{2}\right],
$$

\footnotetext{
6 The case $\lambda \rightarrow 0^{-}$is similar.

7 Since the action $S_{E}$ is parity symmetric, and $\bar{X}$ is parity odd, $W(\lambda)=-W(-\lambda)$, and $V(\bar{X})=V(-\bar{X})$. So, $-v$ will also be a minimum, but we simply pick one of them.

8 The same result is obtained when one defines the effective potential in the region $|\bar{X}|<|v|$ using the Maxwell construction [15].

9 The following is a clarification of an argument presented in [1]
} 
hence

$$
\begin{aligned}
e^{-W} & =(V b)^{-1 / 4} \mathcal{I}(q) \\
\mathcal{I}(q) & =\int_{-\infty}^{\infty} d x \exp \left[-\frac{1}{4} x^{4}-\alpha q^{2 / 3} x^{2}+i q x\right]
\end{aligned}
$$

where

$$
q=\lambda\left(V^{3} / b\right)^{1 / 4} ; \quad \alpha=\frac{1}{2} a\left(\lambda^{2} b\right)^{-1 / 3} .
$$

The integral $\mathcal{I}$ is evaluated in the Appendix, we find, for small $|\lambda|$,

$$
\begin{aligned}
\mathcal{I} & \simeq z e^{-V\left[a^{2} /(4 b)+\lambda^{2} /(4 a)+\cdots\right]} \cos (V|\lambda| \sqrt{|a| / b}+\phi) ; \quad|\lambda| \ll 1, a<0 \\
& \simeq z e^{-V\left[a^{2} /(4 b)+|\lambda| \sqrt{a / b}+\lambda^{2} /(4 a)+\cdots\right]} ; \quad|\lambda| \ll 1, a>0
\end{aligned}
$$

where $z, \phi$ are constants.

For the case of no spontaneous symmetry breaking $(a>0)$ the free energy $F$ is well defined and satisfies $(6)$ though it is not analytic in $\lambda$. When the symmetry is spontaneously broken the free energy suffers from the ailments described in [2]. Note that the bound (6) is satisfied in either case.

Since the free energy is ill defined when $a<0$ (though (6) is still obeyed), the VW argument is incomplete in this case. The problem is that the partition function oscillates, corresponding to a complex $W$; we now argue that this will not occur in any physical system. Indeed, a reasonable requirement for a system is that the Hamiltonian be an additively renormalizable operator that is bounded from below. In this case $\exp (-W)$ equals the $\beta \rightarrow 0$ limit of the partition function $Z=\operatorname{Tr} e^{-\beta H}$. For for any finite $\beta$, the standard expression for $Z$ in terms of energy eigenvalues shows that it cannot be negative, provided we assume that the exponential decrease in the contributions from large (renormalized) energy states is sufficient to guarantee convergence of the trace. Systems obeying these restrictions will have $Z>0$, which then implies $\exp (-W) \geq 0$. In such cases the possibility of negative $a$ (or any set of parameters leading to a complex $W$ ), where parity is spontaneously broken, will not be realized.

This same issue was discussed in [3], where it is claimed that $W(\lambda)$, when defined appropriately, has the same dependence on $\lambda$ independent of the sign of $a$. Although we find that this is not the case, the claims of this reference would be validated by (15) provided the free energy is a concave function of $\lambda$, as is indeed the case when $a>0$.

This, however, is not the case $a<0$. If we adopt the prescription of [3] of defining the free energy through an average over the volume, the results are ambiguous. For example the free energy per unit volume,

$$
f=-\lim _{V \rightarrow \infty} \frac{1}{V} \ln \mathcal{I}
$$

would equal $f=a^{2} /(4 b)+\lambda^{2} /(4 a)+\cdots$ provided we define

$$
\lim _{V \rightarrow \infty} \frac{\ln \cos (c V)}{V}=0
$$

for any fixed real constant $c$. In this case the conclusions of [3] follow: we cannot use the $\lambda$ dependence of the free energy to determine the presence or absence of symmetry breaking. However this is an ad-hoc choice: consider $V_{n}=(n+1 / 2) \pi-e^{-u \pi n}$ for a real constant $u$ and a positive integer $n$. Then letting $V=V_{n}$ and taking the infinite volume limit by letting $n \rightarrow \infty$, we find

$$
\lim _{n \rightarrow \infty} \frac{\ln \cos V_{n}}{V_{n}}=i-u
$$

having taken the branch of the logarithm such that $\ln \cos V_{n}=n \pi(i-u)$. With these choices the limit is certainly non-zero. The point is that the large volume limit of $(\ln \mathcal{I}) / V$ is ill defined. ${ }^{10}$ It is possible to define the limit so that the troublesome oscillatory term does not contribute, but it is unclear whether this is the physically correct definition of the free energy.

We conclude that the concerns raised in $[2,3]$ are valid in that the partition function would not be positive definite in a system exhibiting spontaneous parity violation. However, this situation will not arise for systems with a Hamiltonian bounded from below such that the density of states does not grow faster than the exponential of the energy.

${ }^{10}$ For a different choice of the branch cuts the imaginary term would be absent. 


\section{LATTICE CONSIDERATIONS}

In a series of publications[4] Aoki proposed a phase diagram for lattice QCD that is markedly different from the standard case (both quantitatively and qualitatively) for non-zero lattice spacing (all such peculiar effects disappear in the continuum limit). In this scenario the theory has two phases, one where the 3 pions are massless, being the Goldstone bosons produced by the spontaneous breaking of the usual chiral symmetry. In the second phase flavor symmetry is believed to be spontaneously broken down to $U(1)$ generating two Goldstone bosons (for the case of 2 massless quarks) so that the third pion is massive in this phase. The transitions between phases are of the second order so that the third pion mass vanishes smoothly as the phase boundary is approached. In this second phase parity is also spontaneously broken leading to a non zero expectation value of the order parameter $\left\langle\bar{\psi} i \gamma_{5} \psi\right\rangle$.

Though Aoki's original arguments were made within the lattice-regulated theory, the results of Sect. II would be equally applicable to this case[5] provided the various assumptions made are satisfied. Should this be the case, the presence of a parity-violating phase at finite lattice spacing casts doubt on the results of [1], even though the parity-asymmetric phase disappears in the continuum.

The lattice arguments in ref. [4] are supported by two large-N calculations, one for the 2-dimensional Gross-Neveu model[16] and one for 4-dimensional QCD[17]; both are based on finding minima for the effective potential that do not respect parity. In addition there is also some numerical evidence based on the Monte-Carlo evaluation of several observables. We will revisit these calculations in the paragraphs below.

\section{A. The lattice Gross-Neveu model}

This model contains $N$ spinor fields $\psi_{i}$ where the doubling problem is fixed using Wilson fermions. The dynamics is defined by the lattice action (we take a unit lattice spacing)

$$
S=\frac{1}{2} \sum_{i=1}^{N} \overline{\psi_{i}(n)}\left\{\left[\partial \psi_{i}\right](n)+\frac{1}{2} r\left[\square \psi_{i}\right](n)+\left[\sigma(n)-i \pi(n) \gamma_{5}\right] \psi_{i}(n)\right\}+\frac{N}{2 g^{2}} \sum_{n}\left[\sigma(n)^{2}+\pi(n)^{2}\right]
$$

where $g$ is $N$-independent and the derivatives denote the usual lattice operations[19]. In the large $N$ limit and for constant $\sigma$ and $\pi$, the fermionic determinant yields a function which has a minimum for a non-zero value of $\pi$. Even though quantum fluctuations are known to destroy this non-trivial vacuum[20], this calculation does suggest the possibility of the richer phase diagram described in [4].

These results for the Gross-Neveu model do not contradict general argument presented in [1]. This is because this model does not satisfy an important assumption made in deriving the VW result: since the fermions do not couple vectorially, the fermionic determinant associated with (19) is not positive definite. While this model can be used as guidance in obtaining the phase structure of QCD on the lattice, it does not provide a violation of the VW results.

\section{B. Lattice QCD in 4 dimensions at large $N$}

Another example considered by Aoki is the case of the large $N$ limit ( $N$ again denotes the number of fermions) in lattice QCD in 4 dimensions using Wilson's formalism. Denote by $\sigma(n)=\psi(n) \otimes \overline{\psi(n)}$, then, following [4], we consider the effective potential for these operators in the case where they are position-independent in the large $N$ and strong coupling limits. Replacing then

$$
\sigma(n) \rightarrow \sigma e^{i \theta \gamma_{5}}
$$

the effective action for this order parameter is (we take again unit lattice spacing)[17]

$$
S_{\mathrm{eff}}=4 N \Omega\left\{m \sigma \cos (\theta)-\ln (\sigma)-2\left[\sqrt{1-4 \sigma^{2} \sin (\theta)^{2}}-1-\ln \left(\frac{1+\sqrt{1-4 \sigma^{2} \sin (\theta)^{2}}}{2}\right)\right],\right\}
$$

where $\Omega$ denotes the lattice volume, $m$ the bare fermion mass, and we have chosen the Wilson fermion parameter $r=1$. The effective potential is minus the above expression. We will also replace $N \ln \sigma=(N / 2) \ln \sigma^{2}$ and avoid a spurious imaginary contribution to $S_{\text {eff }}$. This expression has a series of local extrema at the points

$$
\text { (i) : } \cos \theta= \pm 1, \sigma= \pm 1 / m . \quad(\text { ii }): \cos \theta= \pm m \sqrt{\frac{3}{16-m^{2}}}, \sigma= \pm \sqrt{\frac{3}{16-m^{2}}} .
$$


Points (ii) are local minima of $S_{\text {eff }}$ for $m^{2}<4$ but are saddle points for $m>2$; (ii) are saddle points for $m^{2}<16$ and unphysical otherwise. It is remarkable that the curvature of the effective potential at points $(i i)$ is positive in the $\theta$ direction, corresponding to a positive pion mass parameter.

None of these points, however, represent the global minimum of the effective action. In fact we have the behavior,

$$
\begin{aligned}
S_{\mathrm{eff}} & \rightarrow+\infty, \quad \sigma \rightarrow 0, \\
& \rightarrow-\infty, \quad \sigma \rightarrow \infty, \quad \theta=\pi .
\end{aligned}
$$

Hence points $(i)$ represent meta-stable states where parity is broken, the stable vacuum at $\sigma=0$ is parity even. Note that the stability of the $\sigma=0$ vacuum is determined solely by the term $\ln \sigma$ and may be altered by finite $N$ corrections.

This still does not explain why the numerical evaluation of $\left\langle\bar{\psi} \gamma_{5} \psi\right\rangle$ yields a non-zero result. This is understood by the observation [4] that the Wilson action does not lead to a positive fermionic determinant, which follows form the fact that the Wilson term, does not anticommute with $\gamma_{5}$. It is possible to generalize somewhat the results of [13], and to show that the Wilson action leads to a positive fermionic determinant, but only when the fermions carry a real representation of the gauge group [7], which is not the case for QCD. Hence the presence of spontaneous parity violation in QCD with Wilson fermions does not invalidate VW for finite lattice spacing, as the theory does not comply with the assumptions made in [1]. On the other hand, the lattice results do indicate that $\left\langle\bar{\psi} \gamma_{5} \psi\right\rangle \rightarrow 0$ in the continuum limit, as demanded by VW. In this sense the results in [4] support those of [1].

\section{FERMIONIC ORDER PARAMETERS}

In a calculation related to Aoki's, Sharpe and Singleton[5] considered the case of QCD with two flavors to which they added a source for a parity-violating order-parameter. Then they studied the vacuum expectation value of this order parameter as the source strength was set to zero. The fermionic part of the Lagrangian in Euclidean space is

$$
\mathcal{L}_{\text {ferm }}=\bar{\Psi}\left(\not D_{E}+m+h e^{-i \theta \gamma_{5} \tau_{3}}\right) \Psi
$$

where $\Psi$ denotes the fermion iso-doublet (assumed degenerate in mass), and $h$ the source strength. The Euclidean covariant derivative operator $\not_{E}$ is anti-Hermitian (in our conventions the Euclidean gamma matrices are Hermitian[10]), and the Pauli matrix $\tau_{3}$ acts on the isospin variables.

The possibility of spontaneous parity violation can be studied considering the expectation values of operators of the form

$$
\mathcal{O}_{\Gamma}^{(a)}=\overline{\Psi^{a}} \Gamma \Psi^{a}, \quad a=1,2 ;
$$

where $a$ labels the isospin components and $\Gamma$ is a linear combination of the unit matrix, $i \gamma_{5}, \tau_{3}$ and $i \tau_{3} \gamma_{5}$. To obtain the general form of the expectation value,

$$
\left\langle\mathcal{O}_{\Gamma}^{(a)}\right\rangle=\operatorname{Tr}\left\{\Gamma \frac{1}{\not D_{E}+m+h \exp \left[-i \theta \gamma_{5}\left(\tau_{3}\right)_{a a}\right]}\right\},
$$

(no sum over $a$ ) we follow $[5,21]$ and consider the above system in a finite volume; we then expand the above trace using the eigenfunctions of $\not_{E}$ which we denote by $\varphi_{n}$. Explicitly,

$$
\not D_{E} \varphi_{n}=i \lambda_{n} \varphi_{n}
$$

where we used the fact that the Euclidean Dirac operator is anti-Hermitian. As usual [22] we will treat the zero modes separately.

When $\lambda_{n} \neq 0$ the mode $\varphi_{n}$ is paired with $\gamma_{5} \varphi_{n}$ for which

$$
\not D_{E}\left(\gamma_{5} \varphi_{n}\right)=i\left(-\lambda_{n}\right)\left(\gamma_{5} \varphi_{n}\right)
$$

hence in the $\left\{\varphi_{n}, \gamma_{5} \varphi_{n}\right\}$ subspace we have

$$
\not D_{E}+m+h e^{\mp i \theta \gamma_{5}} \rightarrow\left(\begin{array}{cc}
i \lambda_{n}+m+h \cos \theta & \mp i h \sin \theta \\
\mp i h \sin \theta & -i \lambda_{n}+m+h \cos \theta
\end{array}\right),
$$

and we can replace $\gamma_{5} \rightarrow \tau_{1}$ (denoting the usual Pauli matrix). Then the corresponding contributions to the trace (26) are

$$
\operatorname{Tr}\left\{1 \frac{1}{\not D_{E}+m+h e^{\mp i \theta \gamma_{5}}}\right\} \rightarrow \frac{2(h \cos \theta+m)}{\lambda_{n}^{2}+\Lambda^{2}} ; \quad \operatorname{Tr}\left\{\left(i \gamma_{5}\right) \frac{1}{\not D_{E}+m+h e^{\mp i \theta \gamma_{5}}}\right\} \rightarrow \mp \frac{2 h \sin \theta}{\lambda_{n}^{2}+\Lambda^{2}}
$$


where

$$
\Lambda^{2}=h^{2}+m^{2}+2 h m \cos \theta .
$$

For the zero modes $\lambda_{n}=0$ we can choose $\varphi_{n}$ to be an eigenstate of $\gamma_{5}$,

$$
\gamma_{5} \varphi_{n}=\chi_{n} \varphi_{n}, \quad \chi_{n}^{2}=1,
$$

In this one-dimensional subspace we can replace $\gamma_{5} \rightarrow \chi_{n}$ and

$$
\not D_{E}+m+h e^{\mp i \theta \gamma_{5}} \rightarrow h e^{\mp i \theta \chi_{n}}+m .
$$

Then the corresponding contributions to (26) become

$$
\operatorname{Tr}\left\{1 \frac{1}{D_{E}+m+h e^{\mp i \theta \gamma_{5}}}\right\} \rightarrow \frac{h e^{ \pm i \theta \chi_{n}}+m}{\Lambda^{2}} ; \quad \operatorname{Tr}\left\{\left(i \gamma_{5}\right) \frac{1}{\not D_{E}+m+h e^{\mp i \theta \gamma_{5}}}\right\} \rightarrow i \chi_{n} \frac{h e^{ \pm i \theta \chi_{n}}+m}{\Lambda^{2}} .
$$

Adding all terms we find

$$
\begin{aligned}
\operatorname{Tr}\left\{1 \frac{1}{\not D_{E}+m+h e^{\mp i \theta \gamma_{5}}}\right\} & =\sum_{n} \frac{h \cos \theta+m}{\lambda_{n}^{2}+\Lambda^{2}} \pm i \nu \frac{h \sin \theta}{\Lambda^{2}} \\
\operatorname{Tr}\left\{\left(i \gamma_{5}\right) \frac{1}{\not D_{E}+m+h e^{\mp i \theta \gamma_{5}}}\right\} & =\mp \sum_{n} \frac{h \sin \theta+m}{\lambda_{n}^{2}+\Lambda^{2}}+i \nu \frac{h \cos \theta+m}{\Lambda^{2}},
\end{aligned}
$$

where the sum is over all modes (with positive negative and zero eigenvalues), and $\nu$ denotes the index of $\not D_{E}$,

$$
\nu=\sum_{n} \chi_{n}
$$

where this sum is over the zero modes only.

Using these expressions we find, for the operators in (24),

$$
\begin{aligned}
\left\langle\mathcal{O}_{1}^{(a)}\right\rangle & =\frac{h \cos \theta+m}{\Lambda \Omega} \sum_{n} \frac{\Lambda}{\lambda_{n}^{2}+\Lambda^{2}}-i\left(\tau_{3}\right)_{a a} \frac{\nu}{\Omega} \frac{h \sin \theta}{\Lambda^{2}} \\
\left\langle\mathcal{O}_{i \gamma_{5}}^{(a)}\right\rangle & =\left(\tau_{3}\right)_{a a} \frac{h \sin \theta}{\Lambda \Omega} \sum_{n} \frac{\Lambda}{\lambda_{n}^{2}+\Lambda^{2}}+i \frac{\nu}{\Omega} \frac{h \cos \theta+m}{\Lambda^{2}},
\end{aligned}
$$

(no sum over $a$ ) where $\Omega$ denotes the space-time volume. In particular,

$$
\left\langle\int d^{4} x \bar{\Psi} i \gamma_{5} \Psi\right\rangle=2 i \nu \frac{h \cos \theta+m}{\Lambda^{2}}
$$

Taking the infinite volume limit and then letting $h$ approach zero, we obtain

$$
\begin{aligned}
\left\langle\int d^{4} x \bar{\Psi} i \gamma_{5} \Psi\right\rangle_{h=0} & =2 i \frac{\nu}{m} \\
& =\frac{i}{8 \pi^{2}} \int d^{4} x \operatorname{Tr}\left[\tilde{F}_{\mu \nu} F_{\mu \nu}\right]
\end{aligned}
$$

The authors of [5] obtain zero instead of the above expression since they did not include the contribution from the zero modes explicitly. Note also that both isospin components contribute equal amounts to the right-hand side of (39). The fact that we obtain a non-vanishing expression in (39) does not imply that parity is broken: this will be true only if this non-zero value survives the integration over the gauge fields. That integral cannot be evaluated, even formally; yet the fact that the right hand side of (39) is purely imaginary allows us to use the argument leading to (6) which implies that, in fact, parity is not broken.

It is worth noting that (39) is consistent with the anomaly equation in Euclidean space provided we assume that there are no massless excitations, so that the volume integral of the divergence of the axial current vanishes. We also note that the corresponding expression for the parity-even bilinear gives

$$
\langle\bar{\Psi} \Psi\rangle_{h=0}=-\int d \lambda \tilde{\rho}_{A}(\lambda) \frac{2 m}{\lambda^{2}+m^{2}}
$$


where $\tilde{\rho}_{A}$ denotes the spectral density per unit four-volume for the operator $\not D_{E}$. This expression reproduces the corresponding result of [5].

If we add to the Lagrangian a term $\theta \operatorname{Tr} F \tilde{F} /(4 \pi)^{2}$ (where $F$ denote the field strength of the gauge field in the fermionic covariant derivative and $\tilde{F}$ the corresponding dual) then (39) can be interpreted as stating that the expectation value on the left-hand side can be compensated by an appropriate shift in $\theta$. For the case of low-energy QCD this is precisely the same result obtained using a chiral Lagrangian: a constant shift in the $\eta^{\prime}$ field can be compensated by a shift in $\theta[23]$.

The expression (39) validates the claims of [1] in that the expectation value is purely imaginary. We also note that (39) is ill defined in the massless case where it has a $1 / h$ singularity. This problem is connected to the fact that the operators under consideration, $\mathcal{O}_{i \gamma_{5}}^{(a)}$ violate both parity and chiral symmetry. We can repeat the calculations for an operator that violates only parity such as

$$
\mathcal{O}_{D}^{(a)}=\overline{\Psi^{a}} i \gamma_{5} \stackrel{\leftrightarrow}{D} \Psi^{a}
$$

In this case we find

$$
\left\langle\mathcal{O}_{D}^{(a)}\right\rangle=-i\left(\tau_{3}\right)_{a a} h \sin \theta \int d \lambda \tilde{\rho}_{A}(\lambda) \frac{2 \lambda}{\lambda^{2}+m^{2}},
$$

that vanishes as $h \rightarrow 0$, but also vanishes when $\theta=0$ when the theory is parity symmetric. For this operator the expectation value is zero at the level of the fermion integral, in contrast with (39).

It is worth pointing out that $\mathcal{O}_{D}^{(a)}$ is not related to $\mathcal{O}_{i \gamma_{5}}^{(a)}$ in (25) through the use of the equations of motion, in fact, if we replace $\not D \rightarrow \not D+i m$ in $(41) \mathcal{O}_{D}^{(a)}$ remains unchanged. This shows that this operator can be eliminated by performing a chiral rotation on the fermion fields, and is then equivalent to a purely bosonic operator proportional to the index of the Dirac operator $\not D_{E}[24]$.

The authors of [5] also consider the behavior of the lattice version of this theory close to the continuum. They find that the leading contributions to the effective potential for the meson field $\Sigma=\sigma+i \boldsymbol{\tau} \cdot \boldsymbol{\pi},\left(\sigma^{2}+\boldsymbol{\pi}^{2}=1\right)$, equals

$$
V_{\text {eff }}(\Sigma)=-c_{1} \sigma+c_{2} \sigma^{2},
$$

with $c_{2}$ approximately constant (independent of $m$ ) while $c_{1}$ is expected to be a linear function of $m$. The crucial issue here is the sign of $c_{2}$ : if $c_{2}<0$ the potential has a minimum at $\sigma^{2}=1$ that corresponds to a parity-symmetric vacuum where the pion field gets no expectation value. If, on the other hand, $c_{2}>0$ parity will be broken ${ }^{11}$. In view of the above discussion that supports the results of Ref. [1], and suggests that the parity-violating vacua of the large-N, strong coupling lattice theory are not absolutely stable, we believe that, in fact, $c_{2}<0$ as well.

\section{PARITY VIOLATION AT FINITE TEMPERATURE}

In [6] it is noted that systems in a heat bath have an available additional time-like vector $n^{\mu}$ (the temperature vector), that can be used to construct $\mathrm{P}$-violating operators that apparently do not acquire the crucial factor of $i$ upon a Wick rotation. An example is the operator $\epsilon^{\alpha \beta \gamma \delta} n_{\delta} \operatorname{Tr}\left[D_{\alpha} F_{\beta \nu} n^{\nu} F_{\gamma \mu} n^{\mu}\right]$.

In order to examine this claim we consider the case of time-dependent field theory at finite temperature. In this case it is well known[25] that in the functional integral exponent, the time integration should be carried along a complex path starting at $-\infty$ and ending at $-\infty-i \beta$. The temperature vector is, in the rest frame for the system, the tangent to this path, and so it is, in general, complex. For the purposes of evaluating the expectation value of an order parameter the path can be taken parallel to the imaginary axis and, as a result, $n^{\mu}$ is purely imaginary. Since all operators in the class mentioned above are odd in $n^{\mu}$, they $d o$ receive a factor of $i$ and will not violate the claims of $[1]$.

\section{COMMENTS AND CONCLUSIONS}

The above calculations provide a re-examination of the original Witten-Vafa result concerning the absence of spontaneous parity violation in theories with only vector-like fermion couplings. We also reviewed the various criticisms of that result and concluded that none of them is strong enough to invalidate the conclusions of [1].

\footnotetext{
${ }^{11}$ In addition, the phase structure suggested by Aoki is reproduced by this model when $c_{2}>0$.
} 
Theories with scalars can evade the VW result in two ways. Firstly some pseudoscalar fields (if present) can acquire an expectation value, but such order parameters do not receive a factor of $i$ when Wick rotated. Secondly, in such theories the fermionic determinant is not necessarily positive definite (e.g. in theories containing solitons.[26]).

In confining and parity-conserving theories containing fermions and gauge bosons, the low-energy excitations are often scalars and fermions (as is the case for QCD). The comments above suggest that the low-energy effective theories for such models can, in principle, violate parity spontaneously. In fact the standard chiral non-linear sigma model[23], with the addition of an electromagnetic mass[27], allows parity-violating vacua for a certain range of parameters. On the other hand, the VW argument implies that there is no parity violation in the underlying theory. Of course, one could simply argue that the VW result for the underlying theory merely requires that the low-energy parameters are such that no parity violation occurs. Still, this situation opens the possibility that there might be some nonperturbative effects that allow such theories to evade the results of VW. An investigation of this possibility lies beyond the scope of the present publication, though we intend to discuss it fully in the near future [7].

Finally, we note that, as briefly mentioned in [1], the same arguments cannot be used to rule out spontaneous $\mathrm{CP}$ violation in theories without scalars as there are $\mathrm{CP}$-violating operators that remain real upon Wick rotation (e.g. $F_{\mu \nu}^{a} F_{\nu \rho}^{b} F_{\rho \mu}^{c} f_{a b c}$ where $F$ denotes the non-Abelian field strength, and $f$ the group structure constants). So, even though it is known that such theories cannot violate CP explicitly,[28] the possibility of their exhibiting spontaneous $\mathrm{CP}$ violation remains.

\section{Acknowledgments}

J.W. would like to thank T. Onogi for his hospitality at the Yukawa Institute of Theoretical Physics where part of this work was complted, and for helpful comments concerning the Aoki phase. This work was supported in part through funds provided by the U.S. Department of Energy under grants DE-FG02-95ER40899 and DE-FG03-94ER40837.

\section{APPENDIX}

In this appendix we evaluate the integral

$$
\mathcal{I}(q)=\int_{-\infty}^{\infty} d x \exp \left[-\frac{1}{4} x^{4}-\alpha q^{2 / 3} x^{2}+i q x\right]
$$

in the limit of large $|\alpha|$. Our strategy will be to construct a differential equation for $\mathcal{I}$ whose solutions can be obtained by a method similar to the WKB approach. The disadvantage of this approach is that the differential equation has solutions that do not correspond to $\mathcal{I}$. In order to extract the relevant ones we will use the following property,

$$
|\mathcal{I}| \leq \int_{-\infty}^{\infty} \exp \left[-\frac{1}{4} x^{4}-\alpha q^{2 / 3} x^{2}\right]=e^{\alpha^{2} q^{4 / 3} / 2} \sqrt{|\alpha| q^{2 / 3}} K_{1 / 4}\left(\alpha^{2} q^{4 / 3} / 2\right) \stackrel{q \rightarrow \infty}{\longrightarrow} \frac{\sqrt{\pi / \alpha}}{2^{1 / 4} q^{1 / 3}}
$$

that follows from the Schwartz inequality.

Integrating by parts, we find (a prime denotes a $q$ derivative)

$$
\mathcal{I}^{\prime \prime \prime}+u \mathcal{I}^{\prime \prime}+v \mathcal{I}^{\prime}+w \mathcal{I}=0
$$

where

$$
\begin{aligned}
u & =-\frac{8}{3} \alpha^{2} q^{1 / 3}+\frac{8 \alpha}{6 \alpha q-\left(27-32 \alpha^{3}\right) q^{7 / 3}} \\
v & =\frac{4}{9} \alpha\left(4 \alpha^{3}-9\right) q^{2 / 3}-\frac{8}{9} \alpha^{2} q^{-2 / 3}-\frac{(224 / 9) \alpha^{3} q^{1 / 3}}{6 \alpha q-\left(27-32 \alpha^{3}\right) q^{7 / 3}} \\
w & =\frac{4}{9} \alpha^{2} q^{-5 / 3}-\frac{4}{3} \alpha q^{-1 / 3}+\frac{16}{27} \alpha^{4} q^{-1 / 3}+\left(\frac{16 \alpha^{3}}{27}-1\right) q-\frac{32}{9} \frac{\alpha^{2}\left(\alpha q^{-2 / 3}+3 q^{2 / 3}\right)}{6 \alpha q-\left(27-32 \alpha^{3}\right) q^{7 / 3}}
\end{aligned}
$$

In the large $q$ limit the solutions for $\mathcal{I}$ can be obtained by using the Ansatz

$$
\mathcal{I}=\exp \left(-y q^{4 / 3}\right)
$$


that solves (46) for large $q$ provided

$$
64 y^{3}-128 \alpha^{2} y^{2}+16 \alpha\left(8 \alpha^{3}-9\right) y+\left(16 \alpha^{3}-27\right)=0
$$

Of the 3 solutions to this equation one is real for all $\alpha$; we call it $y_{0}$. The two others are complex conjugate of each other for $\alpha \lesssim 0.944941$ and real for $\alpha>0.94491$; we denote them by $y_{ \pm}$with the sign associated with the sign of the imaginary part. The asymptotic behavior of these solutions is given in Table I.

\begin{tabular}{|c|c|c|}
\hline solution & $\alpha \rightarrow+\infty$ & $\alpha \rightarrow-\infty$ \\
\hline$y_{0}$ & $\alpha^{2}+\sqrt{2 \alpha}+1 /(8 \alpha)+\cdots$ & $-1 /(4 \alpha)+\cdots$ \\
\hline$y_{+}$ & $-1 /(4 \alpha)+\cdots$ & $\alpha^{2}+i \sqrt{2|\alpha|}+1 /(8 \alpha)+\cdots$ \\
\hline$y_{-}$ & $\alpha^{2}+\sqrt{2 \alpha}+1 /(8 \alpha)+\cdots$ & $\alpha^{2}-i \sqrt{2|\alpha|}+1 /(8 \alpha)+\cdots$ \\
\hline
\end{tabular}

TABLE I: Asymptotic behavior of the solutions to the differential equation (46)

The required solutions should satisfy (45) which implies that the corresponding $y$ should have a positive real part. In this way we find, for large $|\alpha|$

$$
\begin{aligned}
& \mathcal{I} \simeq Z e^{-q^{4 / 3}\left[\alpha^{2}+1 /(8 \alpha)+\cdots\right]} \cos \left(\sqrt{2|\alpha|} q^{4 / 3}+\phi\right) ; \quad \alpha \rightarrow-\infty \\
& \mathcal{I} \simeq Z e^{-q^{4 / 3}\left[\alpha^{2}+\sqrt{2 \alpha}+1 /(8 \alpha)+\cdots\right]} ; \quad \alpha \rightarrow+\infty
\end{aligned}
$$

where $Z, \phi$ are constants. This is the result used in the text.

[1] C. Vafa and E. Witten, Phys. Rev. Lett. 53, 535 (1984)

[2] V. Azcoiti and A. Galante, Phys. Rev. Lett. 83, 1518 (1999) [arXiv:hep-th/9901068]; Nucl. Phys. Proc. Suppl. 83, 947 (2000) [arXiv:hep-lat/9910014]

[3] X. d. Ji, arXiv:hep-ph/0108162.

[4] S. Aoki, Phys. Rev. D 30, 2653 (1984); Prog. Theor. Phys. Suppl. 122, 179 (1996) [arXiv:hep-lat/9509008]; S. Aoki and A. Gocksch, Phys. Lett. B 231, 449 (1989); Phys. Lett. B 243, 409 (1990); Phys. Rev. D 45, 3845 (1992). S. Aoki, T. Kaneda and A. Ukawa, Phys. Rev. D 56, 1808 (1997) [arXiv:hep-lat/9612019].

[5] S. R. Sharpe and R. J. Singleton, Phys. Rev. D 58, 074501 (1998) [arXiv:hep-lat/9804028].

[6] T. D. Cohen, Phys. Rev. D 64, 047704 (2001) [arXiv:hep-th/0101197].

[7] M. B. Einhorn and J. Wudka, in preparation.

[8] D. Kharzeev, R. D. Pisarski and M. H. Tytgat, Phys. Rev. Lett. 81, 512 (1998) [arXiv:hep-ph/9804221]; arXiv:hepph/0012012. D. Kharzeev and R. D. Pisarski, Phys. Rev. D 61, 111901 (2000) [arXiv:hep-ph/9906401].

[9] C. Itzykson and J.-B. Zuber, Quantum field theory (McGraw-Hill; New York; 1980).

[10] P. van Nieuwenhuizen and A. Waldron, Phys. Lett. B 389, 29 (1996) [arXiv:hep-th/9608174]; arXiv:hep-th/9611043. A. Waldron, Phys. Lett. B 433, 369 (1998) [arXiv:hep-th/9702057].

[11] C. Vafa and E. Witten, Nucl. Phys. B 234, 173 (1984).

[12] R. L. Stratonovich, Dokl. Akad. Nauk. S.S.S.R. 115, 1097 (1957) [Sov. Phys. Doklady 2, 416 (1957)]. J. Hubbard, Phys. [13] Rev. Lett. 3, 77 (1959).

[14] C. Vafa and E. Witten, Nucl. Phys. B 234, 173 (1984).

[15] H.E. Stanley, Introduction to phase transitions and critical phenomena. (Oxford University Pres; New York; 1987).

[16] D. J. Gross and A. Neveu, Phys. Rev. D 10, 3235 (1974).

[17] N. Kawamoto and J. Smit, Nucl. Phys. B 192, 100 (1981).

[18] S. Aoki and A. Gocksch, Phys. Rev. D 45, 3845 (1992). R. G. Edwards, U. M. Heller, R. Narayanan and R. L. Singleton, Nucl. Phys. B 518, 319 (1998) [arXiv:hep-lat/9711029]. R. G. Edwards, U. M. Heller and R. Narayanan, Nucl. Phys. B 535, 403 (1998) [arXiv:hep-lat/9802016].

[19] H. J. Rothe, Lattice gauge theories : an introduction (World Scientific; Singapore ; River Edge, N.J.; 1997).

[20] N. D. Mermin and H. Wagner, Phys. Rev. Lett. 17 1133, (1966). P. C. Hohenberg Phys. Rev. 158, 383 (1967). S. R. Coleman, Commun. Math. Phys. 31 259, (1973). S. k. Ma and R. Rajaraman, Phys. Rev. D 11, 1701 (1975).

[21] K. M. Bitar, U. M. Heller and R. Narayanan, Phys. Lett. B 418, 167 (1998) [arXiv:hep-th/9710052]. M. Bitar, Nucl. Phys. Proc. Suppl. 63, 829 (1998) [arXiv:hep-lat/9709086]. 
[22] M. Atiyah and I. Singer, Ann. Math. 87, 484 (1968). A. S. Schwarz, Commun. Math. Phys. 67, 1 (1979). J. S. Dowker, J. Phys. A 11, 347 (1978). T. Eguchi, P. B. Gilkey and A. J. Hanson, Phys. Rept. 66, 213 (1980).

[23] J. Gasser and H. Leutwyler, Nucl. Phys. B 250, 465 (1985).

[24] K. Fujikawa, Phys. Rev. Lett. 42, 1195 (1979); Phys. Rev. D 21, 2848 (1980) [Erratum-ibid. D 22, 1499 (1980)].

[25] Mills, R., Propagators for many-particle systems; an elementary treatment. (New York; Gordon and Breach; 1969) A. J. Niemi and G. W. Semenoff, Annals Phys. 152, 105 (1984). R. L. Kobes, G. W. Semenoff and N. Weiss, Z. Phys. C 29, 371 (1985).

[26] R. Jackiw and C. Rebbi, Phys. Rev. D 13, 3398 (1976).

[27] J. F. Donoghue and A. F. Perez, Phys. Rev. D 55, 7075 (1997) [arXiv:hep-ph/9611331].

[28] W. Grimus and M. N. Rebelo, Phys. Rept. 281, 239 (1997) [arXiv:hep-ph/9506272]. M. B. Einhorn and J. Wudka, Phys. Rev. D 63, 077901 (2001) [arXiv:hep-ph/0007285]. 